\title{
Discovery of Ubiquitous Fast Propagating Intensity Disturbances by the Chromospheric Lyman Alpha Spectropolarimeter (CLASP)
}

M. Kubo ${ }^{1}$, Y. Katsukawa ${ }^{1}$, Y. Suematsu ${ }^{1}$, R. Kano ${ }^{1}$, T. Bando ${ }^{1}$, N. Narukage ${ }^{1}$, R. Ishikawa $^{1}$, H. Hara ${ }^{1}$, G. Giono ${ }^{1}$, S. Tsuneta ${ }^{2}$, S. Ishikawa ${ }^{2}$, T. Shimizu ${ }^{2}$, T. Sakao ${ }^{2}$, A. Winebarger $^{3}$, K. Kobayashi ${ }^{3}$, J. Cirtain ${ }^{3}$, P. Champey ${ }^{4}$, F. Auchère ${ }^{5}$, J. Trujillo Bueno ${ }^{6}$, A. Asensio Ramos ${ }^{6}$, J. Štěpán ${ }^{7}$, L. Belluzzi ${ }^{8,9}$, R. Manso Sainz ${ }^{10}$, B. De Pontieu ${ }^{11}$, K. Ichimoto $^{12,1}$, M. Carlsson ${ }^{13}$, R. Casini ${ }^{14}$, and M. Goto ${ }^{15}$ masahito.kubo@nao.ac.jp 
Received accepted

\footnotetext{
${ }^{1}$ National Astronomical Observatory of Japan, 2-21-1 Osawa, Mitaka, Tokyo 181-8588, Japan

${ }^{2}$ Institute of Space and Astronautical Science, Japan Aerospace Exploration Agency, 3-1-1 Yoshinodai, Chuo-ku, Sagamihara, Kanagawa 252-5210, Japan

${ }^{3}$ NASA Marshall Space Flight Center, ZP 13, Huntsville, AL 35812, USA

${ }^{4}$ University of Alabama in Huntsville, 301 Sparkman Drive, Huntsville, AL 35899, USA

${ }^{5}$ Institut d'Astrophysique Spatiale, CNRS/Univ. Paris-Sud 11, Bâtiment 121, 91405 Orsay, France

${ }^{6}$ Instituto de Astrofísica de Canarias, E-38205 La Laguna, Tenerife, Spain

${ }^{7}$ Astronomical Institute, Academy of Sciences of the Czech Republic, 25165 Ondrejov, Czech Republic

${ }^{8}$ Istituto Ricerche Solari Locarno, CH - 6605 Locarno Monti, Switzerland

${ }^{9}$ Kiepenheuer-Institut für Sonnenphysik, D-79104 Freiburg, Germany

${ }^{10}$ Max-Planck-Institut für Sonnensystemforschung Justus-von-Liebig-Weg $3 \quad 37077$ Göttingen, Germany

${ }^{11}$ Lockheed Martin Solar \& Astrophysics Laboratory, Palo Alto, CA 94304, USA

${ }^{12}$ Hida Observatory, Kyoto University, Takayama, Gifu 506-1314, Japan

${ }^{13}$ University of Oslo, Postboks 1029 Blindern, 0315 Oslo, Norway

${ }^{14}$ High Altitude Observatory, National Center for Atmospheric Research, Post Office Box 3000, Boulder, CO 80307-3000, USA

${ }^{15}$ National Institute for Fusion Science, Toki, Gifu 509-5292, Japan
} 


\begin{abstract}
High cadence observations by the slit-jaw (SJ) optics system of the sounding rocket experiment known as the Chromospheric Lyman Alpha SpectroPolarimeter (CLASP) reveal ubiquitous intensity disturbances that recurrently propagate in one or both of the chromosphere or transition region at a speed much higher than the sound speed. The CLASP/SJ instrument provides a time series of 2D images taken with broadband filters centered on the Ly $\alpha$ line at a 0.6 s cadence. The fast propagating intensity disturbances are detected in the quiet Sun and in an active region, and at least 20 events are clearly detected in the field of view of $527^{\prime \prime} \times 527^{\prime \prime}$ during the 5 -minute observing time. The apparent speeds of the intensity disturbances range from 150 to $350 \mathrm{~km} \mathrm{~s}^{-1}$, and they are comparable to the local Alfvén speed in the transition region. The intensity disturbances tend to propagate along bright elongated structures away from areas with strong photospheric magnetic fields. This suggests that the observed propagating intensity disturbances are related to the magnetic canopy structures. The maximum distance traveled by the intensity disturbances is of about $10^{\prime \prime}$, and the widths are a few arcseconds, which is is almost determined by the pixel size of $1^{\prime \prime} .03$. The timescale of each intensity pulse is shorter than $30 \mathrm{~s}$. One possible explanation of the fast propagating intensity disturbances observed by CLASP is magnetohydrodynamic fast mode waves.
\end{abstract}

Subject headings: Sun: chromosphere — Sun: transition region - magnetic reconnection - waves 


\section{Introduction}

The solar chromosphere is well known to be very dynamic, and chromospheric waves and jets are commonly observed. A commonly observed dynamic feature in the quiet chromosphere is a thin, jet-like structure called a spicule. A typical speed of longitudinal motions of spicules is $25 \mathrm{~km} \mathrm{~s}^{-1}$, approximately the sound speed of the chromosphere, and their typical lifetime is several minutes up to 15 minutes (e.g., Beckers 1968). Chromospheric events with supersonic motions and/or high frequencies are more frequently observed in non-flaring regions as the spatial and temporal resolutions of the observations improve. Short-lived, high speed spicules called Type II spicules (de Pontieu et al. 2007) have been detected near the solar limb in broadband Ca II H filter images from the Solar Optical Telescope (SOT; Tsuneta et al. 2008) on board Hinode (Kosugi et al. 2007). On the solar disk, absorption features in the blue wings of the Ca II $854 \mathrm{~nm}$ or $\mathrm{H} \alpha 656 \mathrm{~nm}$ lines are often observed with ground-based imaging spectroscopic facilities, and they are called Rapid Blueshifted Excursions (RBEs: Langangen et al. 2008; Rouppe van der Voort et al. 2009). The RBEs and Type II spicules have similar properties (e.g, Pereira et al. 2012; Sekse et al. 2012, 2013): a typical lifetime of $5-150 \mathrm{~s}$, a typical transverse motion of $0-20 \mathrm{~km} \mathrm{~s}^{-1}$, and a typical rising motion of $40-125 \mathrm{~km} \mathrm{~s}^{-1}$. More extreme high-speed events exceeding the Alfvén speed (up to $5000 \mathrm{~km} \mathrm{~s}^{-1}$ ) have been reported near the solar limb (Judge et al. 2012).

In sunspot penumbrae, fine scale jet-like structures called penumbral micro-jets are ubiquitously observed in the lower chromosphere, and their apparent rising motion is faster than $100 \mathrm{~km} \mathrm{~s}^{-1}$ (Katsukawa et al. 2007). Similar chromospheric jet-like structures with supersonic velocities have been intermittently and recurrently observed along a light bridge of a mature sunspot (Shimizu et al. 2009) or a developing sunspot (Toriumi et al. 2015a). The common physical mechanism proposed to explain the fine scale jet-like structures in sunspots is magnetic reconnection between anti-parallel components of relatively vertical and horizontal magnetic field lines (e.g. Ryutova et al. 2008; Sakai \& Smith 2008; Nakamura et al. 2012; Toriumi et al. 2015b).

Quasi-periodic intensity changes with high propagation speeds in the chromosphere have been 
detected by recent high spatial and temporal resolution observations, and they are interpreted as various modes of magnetohydrodynamic (MHD) waves in the chromosphere (see review by Jess et al. 2015). Oscillatory phenomena at high propagation speeds $\left(>100 \mathrm{~km} \mathrm{~s}^{-1}\right)$ and short periods $(<60 \mathrm{~s})$ have been detected along spicules (He et al. 2009; Okamoto \& De Pontieu 2011). Quasi-periodic propagating intensity disturbances with propagation speeds of $50-325 \mathrm{~km} \mathrm{~s}^{-1}$ and periods of about 2 minutes have been observed in $\mathrm{H} \alpha$ along fibrils in the quiet chromosphere (Morton et al. 2012). They suggest that the fast propagating intensity disturbances correspond to fast MHD sausage waves because the intensity and the width of the disturbances are out-of-phase. Small-scale highly dynamic bright blobs are also observed in $\mathrm{H} \alpha$ with propagation speeds in the range of 45 - $111 \mathrm{~km} \mathrm{~s}^{-1}$ along magnetic threads, in both filaments and fibrils related to active regions (Lin et al. 2012). In the cluster of propagating bright blobs, a cascade-like behavior and corresponding bidirectional motions are also observed (Scullion et al. 2015). An MHD fast-mode pulse has been suggested to explain the formation of the propagating bright blob.

In this study, we investigate chromospheric high-speed, short-lived phenomena by using images from the slit-jaw (SJ) optics system of the sounding rocket experiment known as the Chromospheric Lyman Alpha SpectroPolarimeter (CLASP; Kano et al. 2012; Kobayashi et al. 2012). CLASP was launched on September 3, 2016 and observations were obtained for 5 minutes during the flight of about $500 \mathrm{~s}$. The CLASP/SJ obtained a unique data set of chromospheric images with sub-second temporal resolution under seeing free conditions. Faint intensity disturbances propagating at very high speeds are detected in the CLASP/SJ images.

\section{Observations and Data analysis}

\subsection{CLASP/SJ}

A major science target of the CLASP experiment is to explore magnetic fields in the upper chromosphere and the transition region of the quiet Sun. The field-of-view of the CLASP/SJ mainly covered a quiet region, although an active region (NOAA 12405) was included in the field 
of view as a marker for the collaborative observations with other space-borne or ground-based telescopes. The CLASP/SJ successfully obtained a time series of Ly $\alpha$ images at the south-east limb, as shown by the solid box in Figure $1 a$, for about $280 \mathrm{~s}$ with a cadence of $0.6 \mathrm{~s}$. The size of the field of view is $527^{\prime \prime} \times 527^{\prime \prime}$ with a pixel scale of $1^{\prime \prime} 03$. The black vertical line around the center of CLASP/SJ image visualizes the spectropolarimeter's slit (Figure 1b). The CLASP/SJ image was obtained with filters centered on the core of the hydrogen Ly $\alpha$ line $(121.6 \mathrm{~nm})$ and with a full width at half maximum (FWHM) of about $3.5 \mathrm{~nm}$. The center of the Ly $\alpha$ line originates in the chromosphere - corona transition region, but the intensity of the CLASP/SJ is contaminated by emission from the lower chromosphere because of the broad bandwidth. Figure 1 shows that the CLASP/SJ image looks very similar to He II $304 \AA$ images of the Atmospheric Imaging Assembly (AIA; Lemen et al. 2012) on board the Solar Dynamics Observatory (SDO; Pesnell et al. 2012). This means that the intensity of the CLASP/SJ mainly originates from the middle or upper chromosphere.

A standard calibration procedure for imaging data is applied to the time series of the CLASP/SJ images: (1) dark subtraction, (2) flat fielding, (3) gain correction, and (4) removal of image motion. A dark current image is made by using 20 dark images taken just before opening the telescope door and 20 dark images taken just after closing it. The median of the 40 dark images is estimated at each pixel, and then it is subtracted from the original CLASP/SJ images. The flat field map was obtained in the laboratory before the flight when the CCD was almost uniformly illuminated by a Deuterium lamp. The gain of the CCD was also obtained in the laboratory before the flight (Champey et al. 2014, 2015). An image cross-correlation technique was used to align the time series of the CLASP/SJ images to the first image. Images from 17:03:41UT to 17:08:20UT are used since they are taken under stable pointing conditions. The maximum drift of the images is about $1^{\prime \prime}$ during this period and the residual jitter after subtraction of the drift is less than $0 ! 15$ (peak-to-valley). The drift due to the solar rotation is negligible in this study because the solar rotation for 5 minutes is smaller than one pixel of the CLASP/SJ.

In order to catch the high frequency components of solar features, a slow-varying component 
was removed by the following three steps: (1) calculate a running temporal average of 50 images corresponding to $30 \mathrm{~s}$ at each pixel by using the IDL "smooth' function, (2) remove the the running temporal average from a time profile at each pixel in the original images, and (3) calculate a running temporal average of 8 images. The purpose of step 3 is to increase the $\mathrm{S} / \mathrm{N}$ and to reduce the intensity fluctuations due to the rotating waveplate that is located in front of the slit. A complete rotation of the waveplate takes $4.8 \mathrm{~s}$, which corresponds to the duration of 8 images. The intensity in the CLASP/SJ images is slightly modulated by the rotation of the waveplate and a shift of the images is also caused by the wedge or tilt of the rotating waveplate. The image motions are mainly removed by the image cross correlation, and then the integration for one rotation of the waveplate reduces the residual image motions. Hereafter, the image after subtraction of the slow-varying component is called "high-pass-filtered" image. For the high-pass-filtered images, the first and final 40 images are not used because they are affected by the edges in the time domain.

\subsection{Solar Dynamics Observatory}

SDO/AIA provides full disk images of the Sun with several wavelength bands. AIA images in the $304 \AA$ and $171 \AA$ channels are selected for this analysis. The $304 \AA$ and $171 \AA$ channels are dominated by He II $(\log (T)=4.7)$ and Fe IX/X $(\log (T)=5.8 / 6.0)$, respectively. The $304 \AA$ channel is sensitive to plasma in the chromosphere or the transition region, and the $171 \AA$ channel is sensitive to plasma in the quiet corona or the upper transition region. Their cadence is $12 \mathrm{~s}$ and the plate scale is $0^{\prime \prime} 6$. The field-of-view of the CLASP/SJ is determined by cross correlating CLASP/SJ images with AIA $304 \AA$ images. In the case of high-pass-filtered AIA images, a running temporal average of 3 images corresponding to $36 \mathrm{~s}$ is removed from the original time profile at each pixel. Full disk photospheric line-of-sight magnetograms provided by the Helioseismic and Magnetic Imager (HMI; Scherrer et al. 2012) on board SDO are also used in this study. The cadence is $45 \mathrm{~s}$ and the pixel sampling is 0.5 . The line-of-sight magnetogram of the HMI shows that active region NOAA 12405 is a simple bipolar region without any clear sunspots (Figure $1 d$ ). Small magnetic concentrations have a wide distribution in the quiet Sun. 


\subsection{Interface Region Imaging Spectrograph}

The Interface Region Imaging Spectrograph (IRIS; De Pontieu et al. 2014) observed near the center of the field-of-view of CLASP from 16:29UT to 18:50UT. Very large, sparse, 16-step rasters with a step size of $1^{\prime \prime}$ were performed and slit-jaw images (SJIs) in the $1400 \AA$ filter were obtained. We use the Level-2 data of SJIs in this study. The IRIS/SJIs in the $1400 \AA$ filter are dominated by emission from Si IV transition region lines (1394/1403 $\AA$ ). The cadence is 4 - 5 s, and the pixel sampling is $0{ }^{\prime \prime} 16 \times 00^{\prime \prime} 33$. IRIS performed two pointings alternatively along the slit of CLASP, as shown by the dotted boxes in Figure $1 b$, in order to have overlaps between their slits as much as possible. As a result, the IRIS/SJIs outside the overlap between the two pointings have a time gap. In this study, we focus on the overlapping area whose size is $172^{\prime \prime} \times 72^{\prime \prime}$ (see the overlap of the two dotted boxes in Figure 1b). When we make the high-pass-filtered IRIS/SJIs, a time series of IRIS/SJIs at a constant cadence of $4.8 \mathrm{~s}$ is produced by a linear interpolation and then a running temporal average of 6 images is removed from the original time profile at each pixel.

\section{Results}

The time series of original CLASP/SJ images (Animation 1a) shows no significant activity in the chromosphere over the whole field-of-view for the observing period. However, as a result of the removal of slow varying components (Animation 1b), many fast-varying intensity disturbances appear in the active region. Similar disturbances can be seen even in the quiet regions. We find that some of the intensity disturbances recurrently appear and then move in one direction with a speed much higher than the typical sound speed in the chromosphere. Three examples of these intensity disturbances, including one that occurred in the overlapping field of view with IRIS, are described in detail below. Finally, properties of 20 events are summarized. 


\subsection{Case 1: Edge of the active region}

The first example takes place near the edge of the active region in Box 1 of Figure $1 b$. The intensity disturbances appear and move along a bright elongated structure from the active region, as shown by the solid line in Figure 2. There is no strong magnetic field in the photosphere along the solid line but a magnetic concentration is located just outside the upper edge of the solid line. This magnetic concentration is part of the active region. Figure 3 shows the temporal change of the CLASP/SJ intensity and AIA intensity along the solid lines in Figure 2. The white-dark patterns move away from the active region at about $300 \mathrm{~km} \mathrm{~s}^{-1}$ in the high-pass-filtered SJ image (Figure $3 b$ ). Such moving patterns appear at least 4 times. The timescale of each intensity disturbance, i.e., the width of the white pattern in time direction in Figure $3 b$, is less than $20 \mathrm{~s}$. Very weak intensity enhancements moving away from the active region can be also identified in the original SJ intensity (arrows in Figure $3 a$ ). Two groups of propagating intensity disturbances can be seen: the first group is observed in the $\mathrm{t}=0-80 \mathrm{~s}$ time range, and the second group is observed in the $\mathrm{t}=100-230 \mathrm{~s}$ time range. In each group, two propagating intensity disturbances sequentially appear within $60 \mathrm{~s}$. The travel distance of each propagating intensity disturbance is about $10^{\prime \prime}$ and the width is about $2^{\prime \prime}-3^{\prime \prime}$, which is almost same as the width of the bright elongated structure.

The advantage of the high temporal resolution of the CLASP/SJ is obvious when the space-time plots of AIA (Figures $3 d$ and $f$ ) are compared with the ones of the CLASP/SJ. No clear propagating patterns are observed in the space-time plots of AIA. The weak bright features with a longer lifetime are observed in the original AIA $304 \AA$ and AIA $171 \AA$ intensity maps, as shown in the boxes of Figure 3. They may be related with the fast propagating intensity disturbances observed by the CLASP/SJ. 


\subsection{Case 2: Limb in the quiet Sun}

The second example is observed along a bright thread in Box 2 at the limb. This region seems to be a normal quiet region as shown in Figure 4. Although magnetic field structures in the line-of-sight magnetogram are not clear because of the foreshortening effect, the relatively strong positive and negative magnetic concentrations can be seen within the disk in Box 2. The intensity disturbances move outward from the limb at about $200 \mathrm{~km} \mathrm{~s}^{-1}$ in the high-pass-filtered SJ image (Figure $5 b$ ), and they recurrently appear for about 3 minutes. The timescale of each intensity disturbance is less than $20 \mathrm{~s}$. The length of the propagating intensity disturbances is about $5^{\prime \prime}$ or slightly shorter, and their width is comparable to the bright thread with the size of a few pixels. The space-time plots of the high-pass-filtered AIA images show no significant propagating features. Some features slightly brighter than their surroundings are observed for the whole period in the original AIA $304 \AA$ images around the position $\left(\mathrm{x}=10^{\prime \prime}\right)$ where the intensity disturbances are observed by the CLASP/SJ. Such features are not observed there in the original AIA $171 \AA$ images.

We examine a dependence of the intensity disturbances on the temporal window in order to confirm whether such disturbances are artificial or not. Figure 6 shows that the propagating intensity disturbances become less clear as the smoothing width is longer because low frequency components with a strong power spectrum density are more contaminated. However, the fast propagating intensity disturbances can be identified when the smoothing time window is shorter than $90 \mathrm{~s}$. This means that the observed fast propagating intensity disturbances are not artificial.

In this paper, we do not discuss the periodicity of the fast propagating intensity disturbances. The repetition time between each fast propagating intensity disturbance is similar to the temporal window $(30 \mathrm{~s})$ of the smooth function when the running temporal average of the intensity is removed from the original ones. This may be due to the fact that the high-pass filtering enhances the features having temporal frequencies similar to that of the cut-off of the filter (Auchère et al. 2016). Further analysis is required in order to determine whether or not the fast propagating disturbances have a preferential repetition rate, i.e., whether or not they are periodic. 


\subsection{Case 3: Quiet Sun}

The third example is observed in the quiet region and it good overlaps with the field-of-view of the IRIS/SJIs, as shown in Figure 7. A bright structure is observed at the center of the target region in the CLASP/SJ, AIA $304 \AA$, and IRIS/SJ images. A magnetic concentration with negative magnetic polarity is also observed around the center of the target region. The propagating intensity disturbances are detected as shown by the sky-blue arrows in Figure $8 b$, although they are less clear when compared with the previous two examples. Their propagation speeds are about $180 \mathrm{~km} \mathrm{~s}^{-1}$, and the timescale of each intensity disturbance is less than $20 \mathrm{~s}$. One interesting property of this example is that the propagating intensity disturbances with the opposite direction (purple arrows) are also seen in the same region. In particular, the intensity disturbances at $\mathrm{t} \sim 170 \mathrm{~s}$ propagate in two (or more) directions simultaneously at similar speeds. The starting point of the propagating intensity disturbances nearly corresponds to the magnetic concentration with negative polarity.

The space-time plots of the AIA and IRIS images show intensity enhancements around the center of the target region from $\mathrm{t} \sim 100 \mathrm{~s}$. The intensity disturbances at short timescales are observed in the high-pass-filtered IRIS/SJIs as well as the CLASP/SJ image during this period, but the propagation patterns cannot be seen in the high-pass-filtered IRIS/SJIs. One possibility of no detection of the propagation patterns is that the fast propagating intensity disturbances observed with the CLASP/SJ mostly occur in chromospheric structures. However, we cannot distinguish whether the absence of detection of the propagating patterns is actually from solar structures or instrumental effects of the current data sets. The high-pass-filtered IRIS /SJ movie shows the slit, dust, and noise patterns of the CCD move with a slit scanning. Such moving patterns are significant in the movie and they make it difficult to detect the faint propagating patterns. The sit-and-stare observations of IRIS would be better to detect such a faint and fast propagating patterns. The high cadence observations by the IRIS/SJI with multiple filters are important for understating nature of the fast propagating intensity disturbances detected by the CLASP/SJ. 


\subsection{Properties of 20 events}

The intensity disturbances propagating in one direction with speeds much faster than the sound speed in the chromosphere are clearly observed in at least 20 areas as shown by the boxes in Figure $1 b$. These 20 events are manually selected from the time series of the high-pass-filtered CLASP/SJ images. The propagating intensity disturbance that occurred just one time are not discussed here. Thus, the intensity disturbances are recurrently observed at the same position in the selected 20 events. The fast propagating intensity disturbances are observed both in the active region and in the quiet Sun. Only two events (\#19 and \#20) are selected in the active region because intensity disturbances are observed almost everywhere in the active region and the intensity disturbances propagating in different directions are mixed in many cases. This makes it difficult to identify the intensity disturbances propagating in one direction and also to estimate the speed of the disturbances. Hence, we mainly focus on the properties of the fast intensity disturbances in the quiet Sun.

The propagating intensity disturbances tend to be observed in the bright areas rather than in the dark areas in the CLASP/SJ images. The bright areas roughly correspond to network regions with stronger magnetic fields in the photosphere. The amplitude of the intensity disturbances is typically $2 \%-5 \%$ of the averaged original intensity. In the dark areas, the propagating intensity disturbances are, if any, obscured by photon noise. The apparent speeds of the 18 events in the quiet Sun range from $150 \mathrm{~km} \mathrm{~s}^{-1}$ to $350 \mathrm{~km} \mathrm{~s}^{-1}$. The travel distances of the propagation are typically $5^{\prime \prime}-10^{\prime \prime}$. The minimum distance ( $\sim 5$ pixels) is determined by the minimum pixels needed to identify a propagating phenomenon. The widths of the propagating intensity disturbances are not well determined due to lack of spatial resolution, but they are mostly within a couple of pixels. The timescale of the intensity disturbances is less than $30 \mathrm{~s}$. There is no preferable direction of the propagation toward the limb or toward the disk center, at least for the observed events. However, the intensity disturbances tend to propagate away from the areas with the strong photospheric magnetic fields (i.e. active region or network region). The relation between the fast propagating intensity disturbances and activities in the upper atmospheric layers observed with AIA is not 
clear. It is hard for AIA images to detect such phenomena with a high speed and a weak intensity fluctuation.

\section{Summary}

The high cadence observations by the CLASP/SJ reveal that the fast propagating disturbances frequently and ubiquitously appear in the chromosphere or the transition region. Such phenomena are observed both in the quiet Sun and in the active region. The apparent propagation speeds are $150-350 \mathrm{~km} \mathrm{~s}^{-1}$ which are much faster than the typical sound speed in the chromosphere or the transition region. It is comparable to the local Alfvén speed in the transition region with the assumption of $10 \mathrm{G}$ magnetic field strength. Another interesting property of the fast propagating intensity disturbances is that they recurrently happen at the same place for observing period of about 5 minutes. The timescale of our observed intensity disturbances is much shorter than quasi-periodic propagating intensity disturbances with the periods of several minutes that are interpreted as slow propagating magneto-acoustic waves (see review by De Moortel \& Nakariakov 2012).

The fast propagating intensity disturbances as observed by the CLASP/SJ seem to be different from well-known chromospheric jet-like phenomena: spicules, chromospheric anemone jets (Shibata et al. 2007), or RBEs. The speed of these phenomena is mostly less than $100 \mathrm{~km}$ $s^{-1}$, and the CLASP/SJ cannot resolve such fine scale structures. Similar propagating intensity disturbances have been reported in $\mathrm{H} \alpha$ along the fibrils in the quiet chromosphere (Morton et al. 2012). The highest speed of $325 \mathrm{~km} \mathrm{~s}^{-1}$ of such disturbances is very similar to that of the disturbances investigated in this work, but their averaged speed is lower than our intensity disturbances. In addition, the typical period of about 2 minutes is longer than the timescale of our cases. The combination of the high cadence, seeing free conditions, and large field-of-view leads to detect such faint events with high propagation speeds at a shorter timescale more clearly and more ubiquitously. 
The candidates to explain the observed fast propagating intensity disturbances are roughly divided into two groups: (1) MHD waves (oscillations) or (2) repetitive plasma flows. In the case of MHD waves, the intensity fluctuations with the propagations at the Alfvén speed suggest the possibility of a MHD fast mode wave. One issue on the scenario of the MHD fast mode wave is the anisotropic propagations of the intensity disturbances. The intensity disturbances propagate along the bright elongated structures. One example of the propagation in multi directions is reported in Section 3.3 but the isotropic propagation cannot be identified. One possibility is that only part of the fast mode wave can be detected because bright and long structures are needed to catch the faint intensity fluctuations propagating at very high speeds. Another possibility is that the fast propagating intensity disturbances is the fast MHD sausage wave along the magnetic flux tube as proposed by Morton et al. (2012) to explain their detected fast intensity disturbances. On the other hand, if the observed fast propagating intensity disturbances correspond to repetitive plasma flows, they are most probably reconnection jets. Fast and repetitive magnetic reconnection is expected in the transition region to explain the observed properties (corresponding to "'EUV jets/EUV microflares' in Shibata et al. 2007). EUV brightenings called blinkers (Harrison 1997) are often observed with chromospheric counterparts in the quiet Sun and active regions (Brković \& Peter 2003; Brooks et al. 2004). The typical lifetime of blinkers is longer than 5 minutes that is much longer than the timescale of our observed intensity disturbances. The weak intensity enhancements with longer lifetime in AIA $304 \AA$ and $171 \AA$ images are only seen in some events, and the fast propagating intensity disturbances are observed even in the region without any EUV brightenings. The relation between blinkers (or EUV microflares) and our intensity disturbances is not clear. Therefore, the fast propagating intensity disturbances may not always correspond to the plasma flows induced by magnetic reconnection. Note that magnetic reconnection itself is one of candidates for excitation process of MHD waves.

For better understanding the fast propagating intensity disturbances discovered by the CLASP/SJ, high cadence observations of spectral lines emitted in the upper chromosphere or the transition region are important. For example, the investigation of Doppler shifts tells us 
whether the observed propagations of intensity disturbances correspond to plasma motions (flows) or pattern motions (waves). In a next paper, we will investigate a time series of $\operatorname{Ly} \alpha$ spectrum measured at $0.3 \mathrm{~s}$ cadence by the spectropolarimeter of CLASP.

We would like to thank Drs. T. J. Okamoto, P. Antolin, S. Toriumi, T. Yokoyama, and R. Rutten for useful discussions. We gratefully acknowledge the Chromospheric Lyman-Alpha Spectropolarimeter (CLASP) team. The team was an international partnership between NASA Marshall Space Flight Center, National Astronomical Observatory of Japan (NAOJ), Japan Aerospace Exploration Agency (JAXA), Instituto de Astrofísica de Canarias (IAC) and Institut d'Astrophysique Spatiale; additional partners include Astronomical Institute ASCR, Lockheed Martin, and University of Oslo. The slit-jaw optics of CLASP was manufactured by Genesia Corp and we are grateful to Drs. N. Takeyama, Y. Kanai, and Ms. Y. Sakakibara. Japanese participation is funded by the basic research program of ISAS/JAXA, internal research funding of NAOJ, and JSPS KAKENHI Grant Numbers 23340052, 24740134, 24340040, and 25220703. US participation is funded by NASA Low Cost Access to Space (Award Number 12-SHP 12/2-0283). Spanish participation has been funded by the Ministry of Economy and Competitiveness through project AYA2010-18029 (Solar Magnetism and Astrophysical Spectropolarimetry). French hardware participation was funded by Centre National d'Etudes Spatiales (CNES). J. Š. acknowledges the support of the Czech Science Foundation via the grant 16-16861S and by the project RVO:67985815 of the Academy of Sciences of the Czech Republic. 


\section{REFERENCES}

Auchère, F., Froment, C., Bocchialini, K., et al. 2016, ApJ, (in press)

Beckers, J. M. 1968, Sol. Phys., 3, 367

Brković, A., \& Peter, H. 2003, A\&A, 406, 363

Brooks, D. H., Kurokawa, H., Kamio, S., et al. 2004, ApJ, 602, 1051

Champey, P., Kobayashi, K., Winebarger, A., et al. 2014, Proc. SPIE, 9144, 914439

Champey, P., Kobayashi, K., Winebarger, A., et al. 2015, Proc. SPIE, 9601, 96010Z

De Moortel, I., \& Nakariakov, V. M. 2012, Philosophical Transactions of the Royal Society of London Series A, 370, 3193

de Pontieu, B., McIntosh, S., Hansteen, V. H., et al. 2007, PASJ, 59, S655

De Pontieu, B., Title, A. M., Lemen, J. R., et al. 2014, Sol. Phys., 289, 2733

Harrison, R. A. 1997, Sol. Phys., 175, 467

He, J.-S., Tu, C.-Y., Marsch, E., et al. 2009, A\&A, 497, 525

Jess, D. B., Morton, R. J., Verth, G., et al. 2015, Space Sci. Rev., 190, 103

Judge, P. G., Reardon, K., \& Cauzzi, G. 2012, ApJ, 755, L11

Kano, R., Bando, T., Narukage, N., et al. 2012, Proc. SPIE, 8443, 84434F

Katsukawa, Y., Berger, T. E., Ichimoto, K., et al. 2007, Science, 318, 1594

Kobayashi, K., Kano, R., Trujillo-Bueno, J., et al. 2012, Fifth Hinode Science Meeting, 456, 233

Kosugi, T., et al. 2007, Sol. Phys., 243, 3

Langangen, Ø., De Pontieu, B., Carlsson, M., et al. 2008, ApJ, 679, L167 
Lemen, J. R., Title, A. M., Akin, D. J., et al. 2012, Sol. Phys., 275, 17

Lin, Y., Engvold, O., \& Rouppe van der Voort, L. H. M. 2012, ApJ, 747, 129

Morton, R. J., Verth, G., Jess, D. B., et al. 2012, Nature Communications, 3, 1315

Nakamura, N., Shibata, K., \& Isobe, H. 2012, ApJ, 761, 87

Nakariakov, V. M., \& Melnikov, V. F. 2009, Space Sci. Rev., 149, 119

Okamoto, T. J., \& De Pontieu, B. 2011, ApJ, 736, L24

Pereira, T. M. D., De Pontieu, B., \& Carlsson, M. 2012, ApJ, 759, 18

Pesnell, W. D., Thompson, B. J., \& Chamberlin, P. C. 2012, Sol. Phys., 275, 3

Rouppe van der Voort, L., Leenaarts, J., de Pontieu, B., Carlsson, M., \& Vissers, G. 2009, ApJ, 705,272

Ryutova, M., Berger, T., Frank, Z., \& Title, A. 2008, ApJ, 686, 1404-1419

Sakai, J. I., \& Smith, P. D. 2008, ApJ, 687, L127

Scherrer, P. H., Schou, J., Bush, R. I., et al. 2012, Sol. Phys., 275, 207

Scullion, E., Engvold, O., Lin, Y., \& Rouppe van der Voort, L. 2015, ApJ, 814, 123

Sekse, D. H., Rouppe van der Voort, L., \& De Pontieu, B. 2012, ApJ, 752, 108

Sekse, D. H., Rouppe van der Voort, L., \& De Pontieu, B. 2013, ApJ, 764, 164

Shibata, K., Nakamura, T., Matsumoto, T., et al. 2007, Science, 318, 1591

Shimizu, T., Katsukawa, Y., Kubo, M., et al. 2009, ApJ, 696, L66

Toriumi, S., Katsukawa, Y., \& Cheung, M. C. M. 2015, ApJ, 811, 137

Toriumi, S., Cheung, M. C. M., \& Katsukawa, Y. 2015, ApJ, 811, 138 
Tsuneta, S., et al. 2008, Sol. Phys., 249, 167

This manuscript was prepared with the AAS IATEX macros v5.2. 

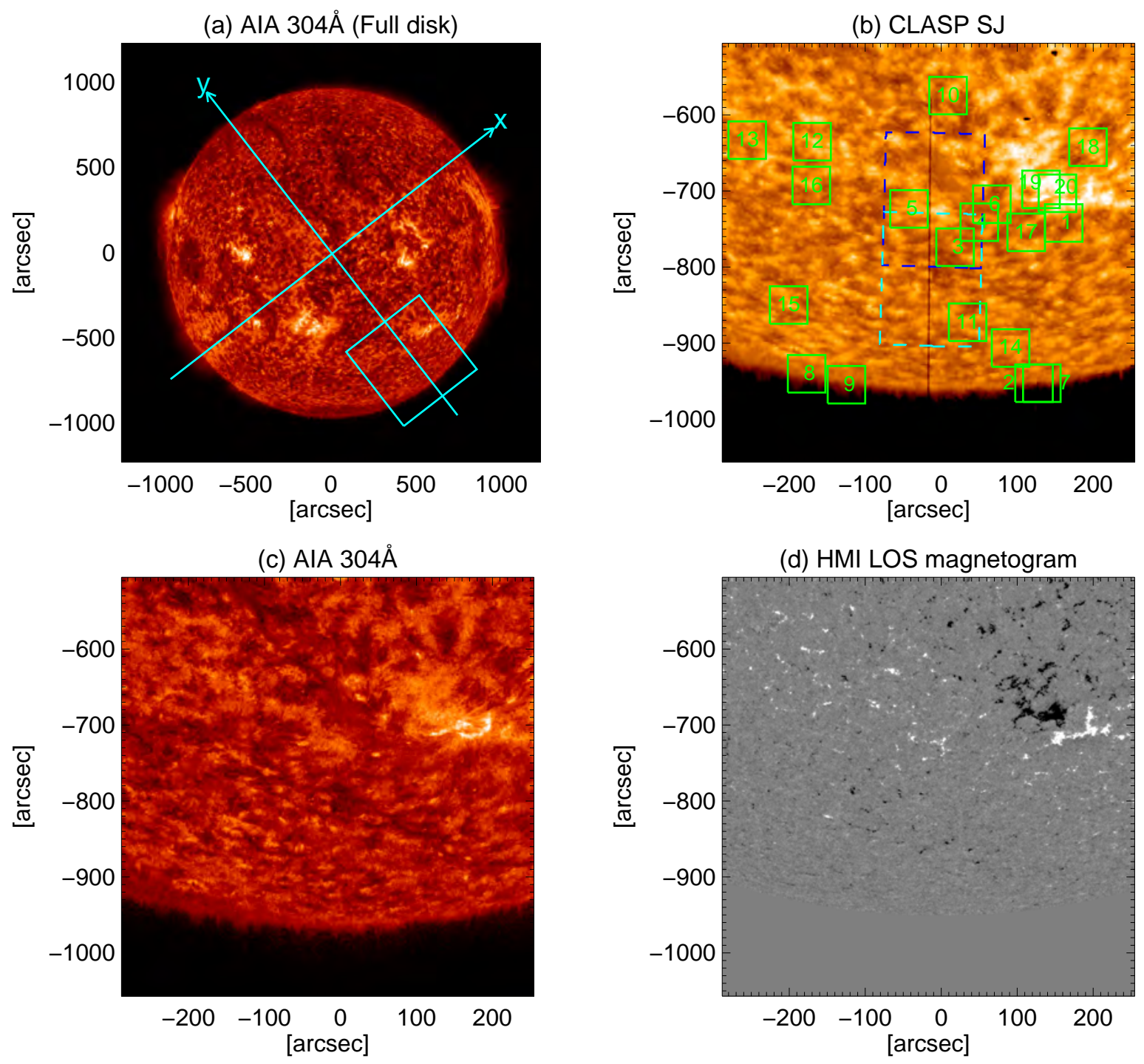

Fig. 1.- (a) SDO/AIA $304 \AA$ image at 17:03:42UT on September 3, 2015. The solid box is identical to the field of view of panels $(b)-(d)$. (b) CLASP/SJ image taken at 17:03:41UT. The positions in the vertical and horizontal axes are given with respect to the center of the solar disk along the $\mathrm{X}$ and $\mathrm{Y}$ axes in panel $(a)$, respectively. Fast propagating intensity disturbances are clearly observed around the center of the green boxes. The two dashed boxes shows the field-of-views of the IRIS/SJIs. (c) SDO/AIA $304 \AA$ image with the same field of view as that of CLASP/SJ. (d) SDO/HMI line-of-sight magnetogram at 17:03:35UT. 

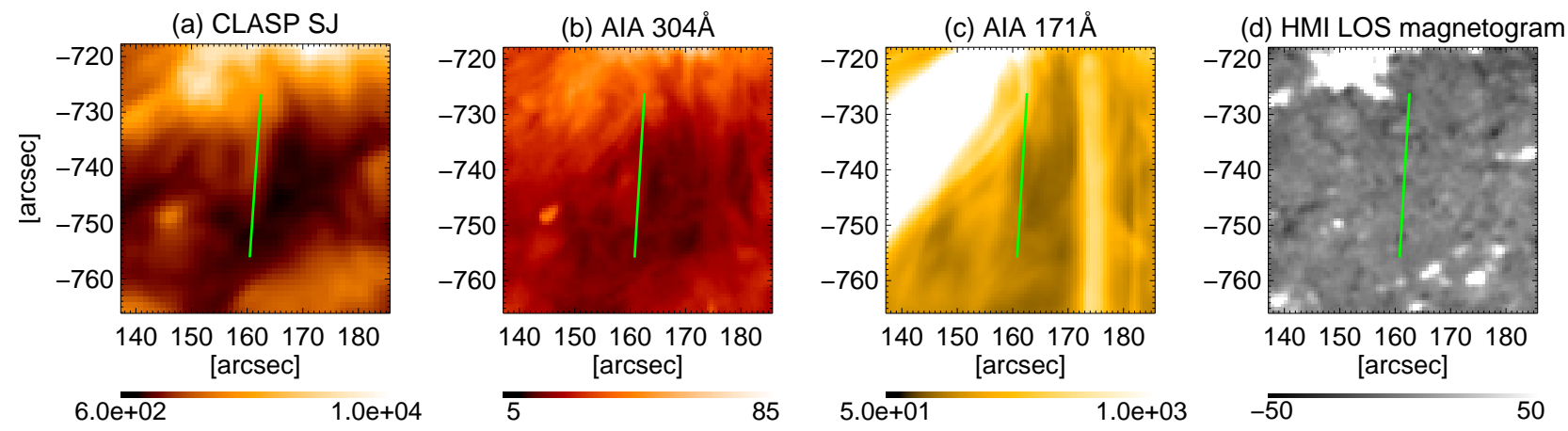

Fig. 2.- (a) CLASP/SJ image, (b) AIA $304 \AA$ image, (c) AIA $171 \AA$ image, and (d) HMI line-of-sight magnetogram in Box 1 of Figure 1b. These images are averaged over the whole period of the limb observations. The CLASP/SJ image, the AIA images, and the HMI magnetogram are in units of the number of electrons, digital number [DN], and gauss, respectively. The solid lines are used to obtain space vs. time plots shown in Fig. 3. 

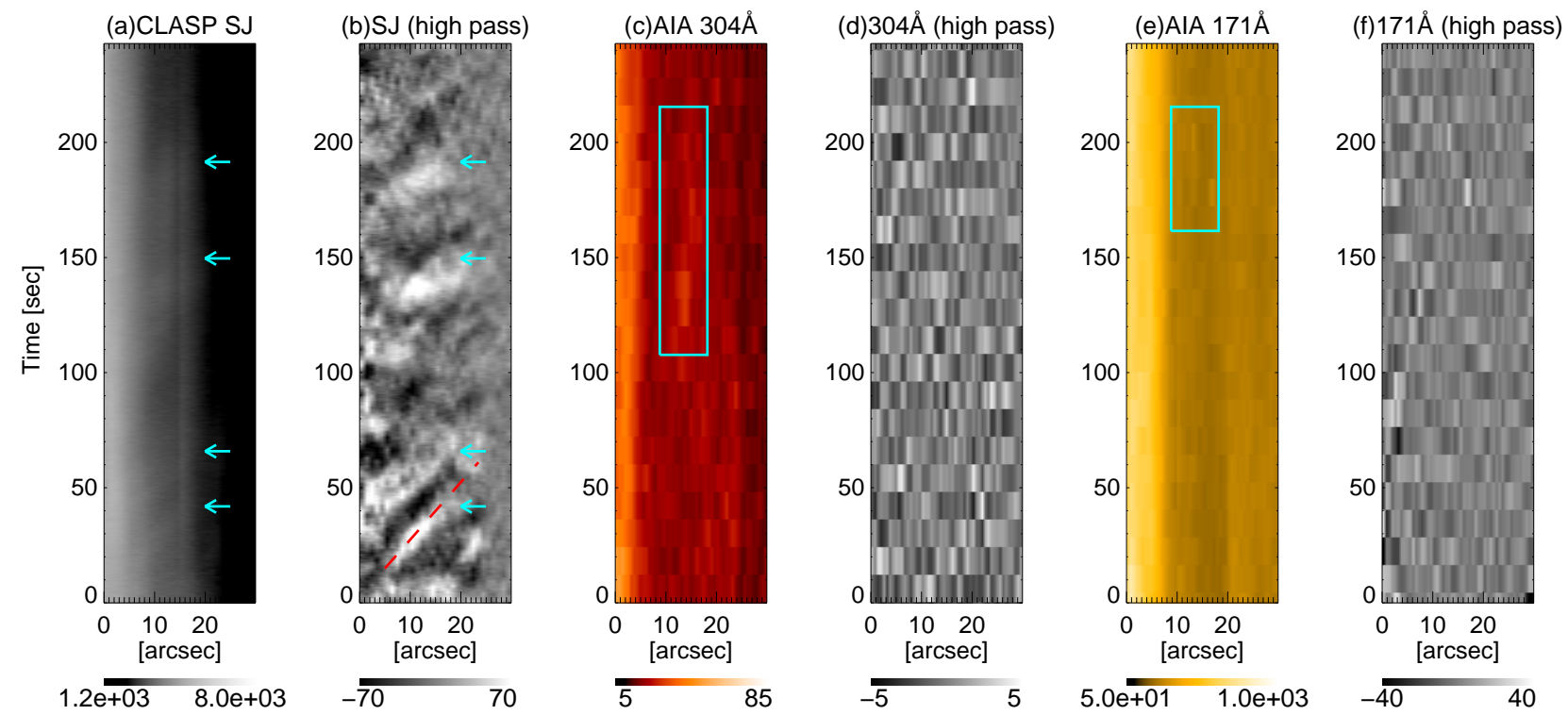

Fig. 3.- Space vs. time plots along the solid lines in Figure 2 for $(a)$ CLASP/SJ image, $(b)$ high-pass-filtered CLASP/SJ image, (c) AIA $304 \AA$ image, $(d)$ high-pass-filtered AIA 304 $\AA$ image, (e) AIA $171 \AA$ image, and $(f)$ high-pass-filtered AIA $171 \AA$ image. The dashed line in panel $(b)$ corresponds to $300 \mathrm{~km} \mathrm{~s}^{-1}$. The zero of the horizontal axis corresponds to the upper edge of the solid line in Figure 2. The zero of the vertical axis corresponds to 17:03:59UT on September 3, 2015.

(a) CLASP SJ

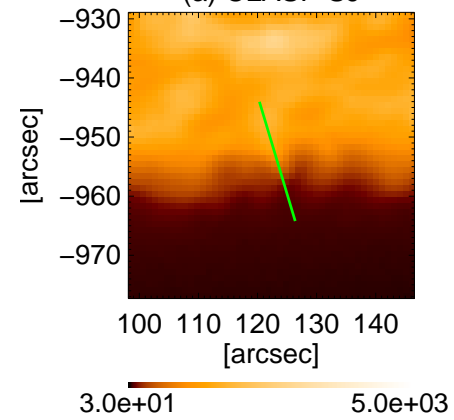

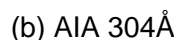

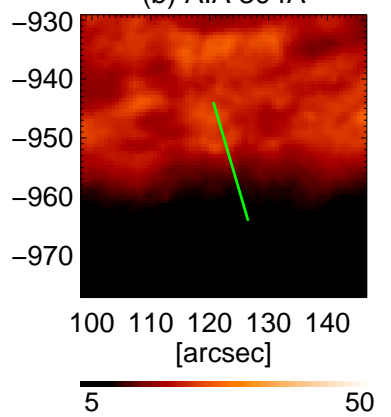

(c) $\mathrm{AIA} 171 \AA$

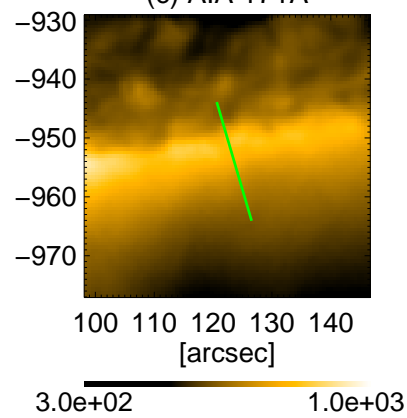

(d) HMI LOS magnetogram

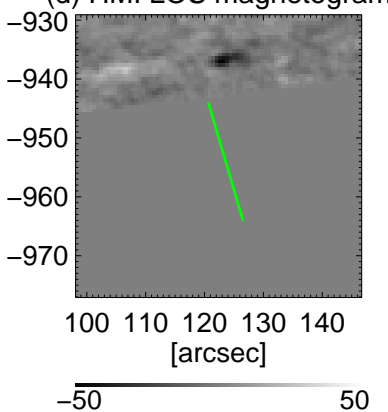

Fig. 4.- Same as Figure 2 but for Box 2 of Figure $1 b$. 

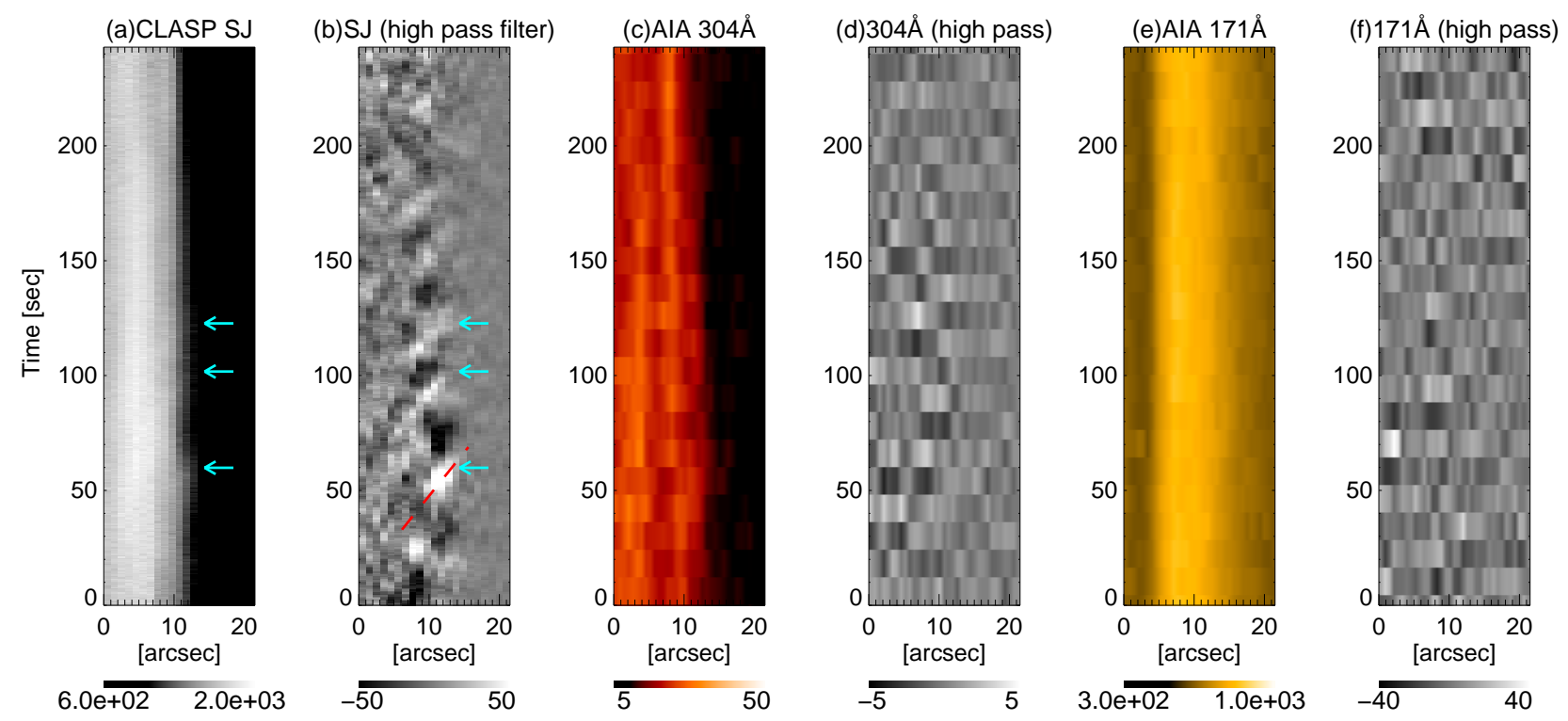

Fig. 5.- Same as Figure 3 but for space vs. time plots along the solid lines in Figure 4. The dashed line in panel $(b)$ corresponds to $200 \mathrm{~km} \mathrm{~s}^{-1}$. The zero of the horizontal axis corresponds to the upper edge of the solid line in Figure 4. 

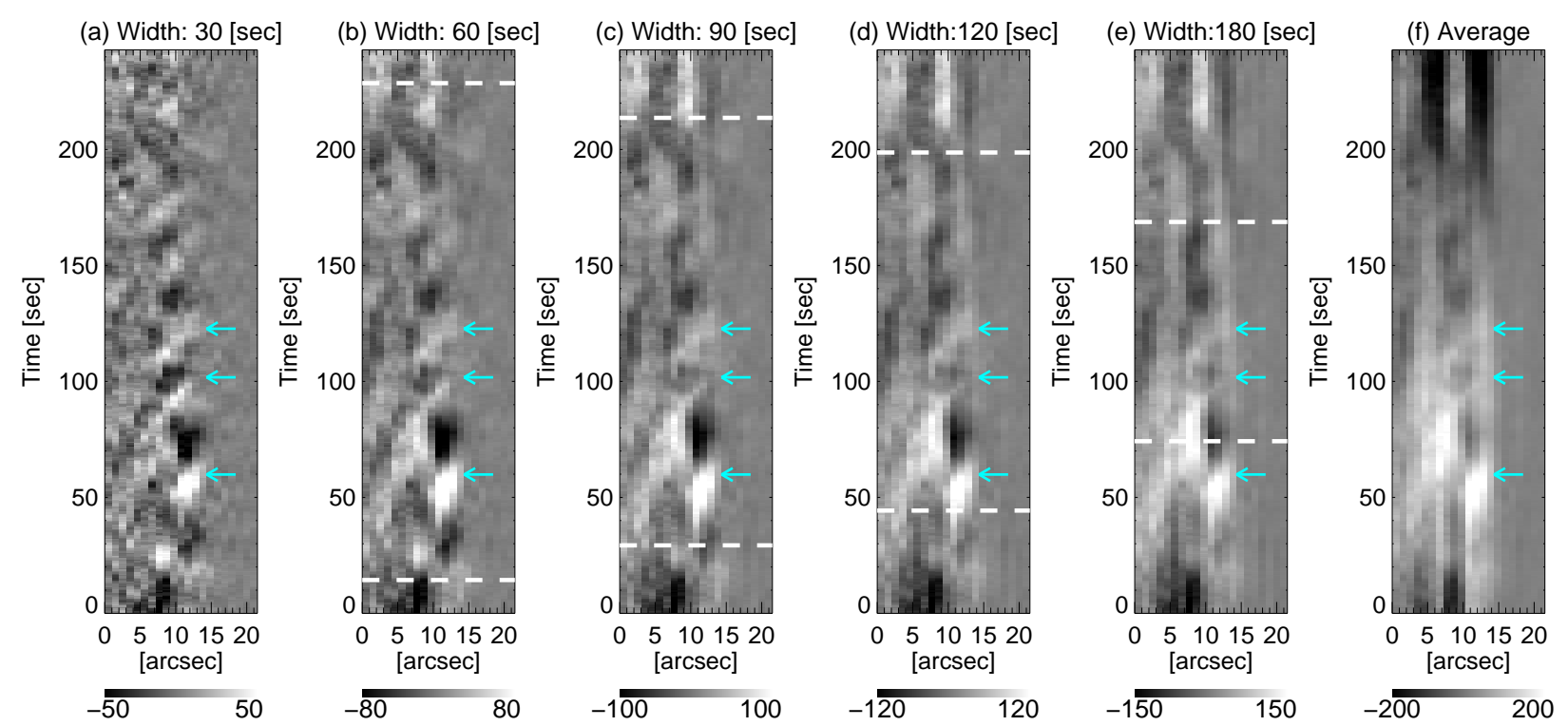

Fig. 6. - Dependence on the width of smoothing window. Panels $(a)-(e)$ show space vs. time plots of the high-pass-filtered CLASP/SJ image with the smoothing window of $30 \mathrm{~s}$, $60 \mathrm{~s}, 90 \mathrm{~s}, 120 \mathrm{~s}$, and $180 \mathrm{~s}$, respectively. Panel $(a)$ is identical to panel $(b)$ in Figure 5. The period between the two horizontal dashed lines is free from the edge treatment of the "smoothing" function. Panel $(f)$ is made by subtraction of the average over the whole observing period from the original CLASP SJ images.

(a) CLASP SJ

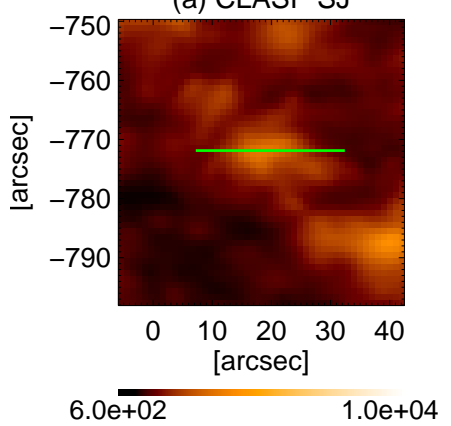

(b) AIA 304^

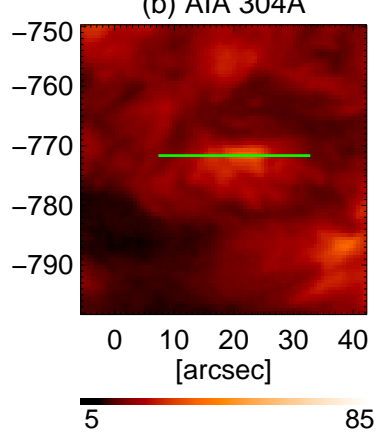

(c) IRS SJI $1400 \AA$

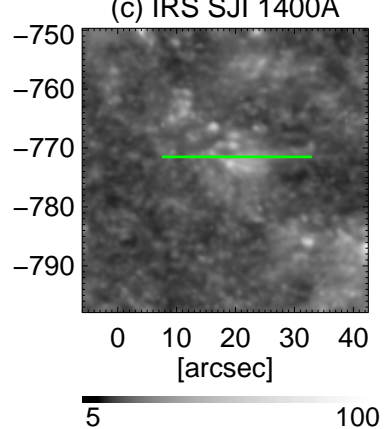

(d) HMI LOS magnetogram

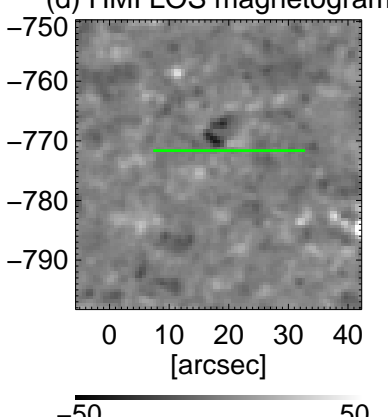

Fig. 7.- Panels $(a),(b)$, and $(d)$ are same as Figure 2 but for Box 3 of Figure $1 b$. Panel (c) is IRIS/SJ $1400 \AA$ image in units of DN. 

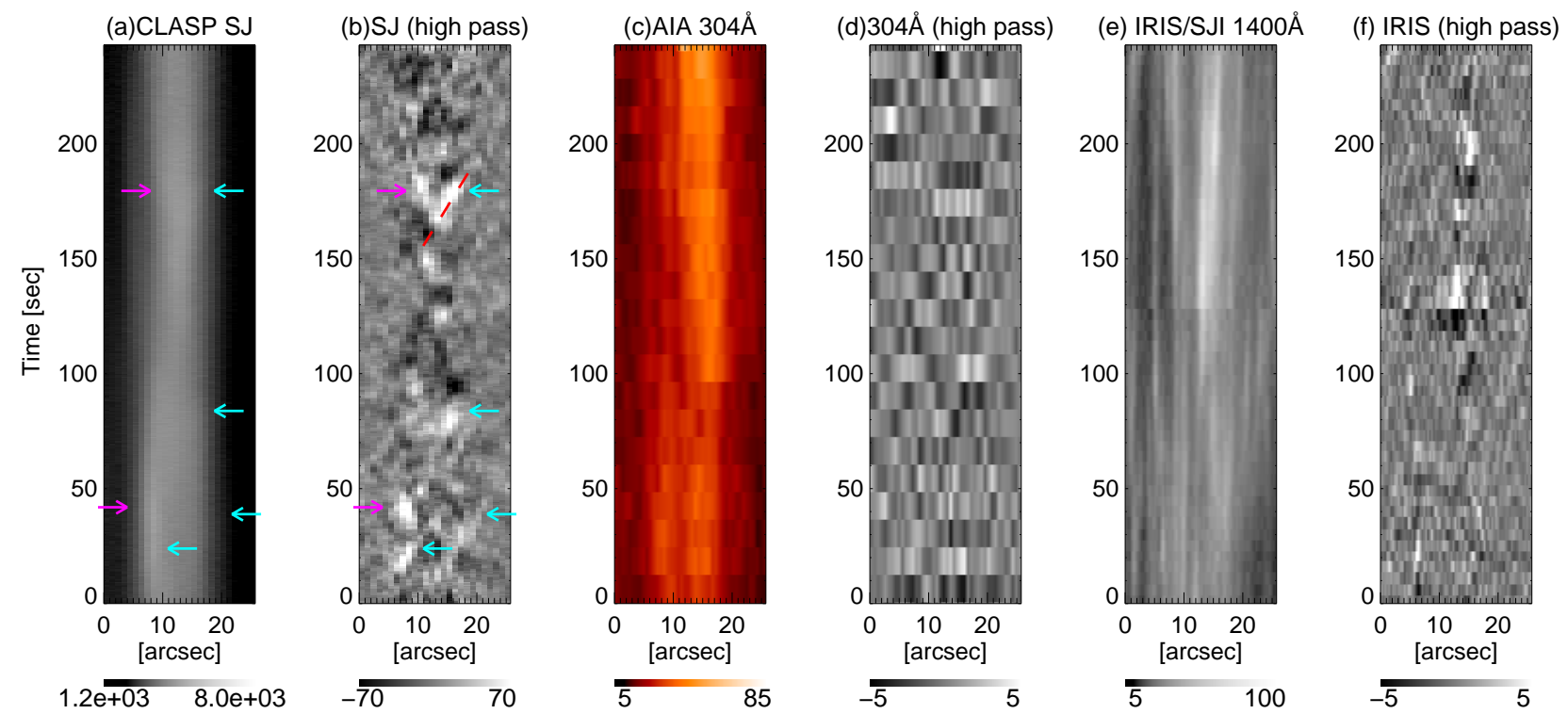

Fig. 8.- Panels $(a)-(d)$ are same as Figure 3 but for space vs. time plots along the solid lines in Figure 7. Panels $(e)$ and $(f)$ are space vs. time plots along the same line for IRIS /SJ $1400 \AA$ image and its high-pass-filtered image, respectively. The dashed line in panel (b) corresponds to $180 \mathrm{~km} \mathrm{~s}^{-1}$. The zero of the horizontal axis corresponds to the left edge of the solid line in Figure 7. 